\title{
Outcomes of Thoracoscopic Thymectomy in Children: Experience in A Resource Challenged Setup
}

\author{
Rengarajan $\mathrm{R}^{1}$,Venkatesa Mohan $\mathrm{N}^{2}$,Vijaya Raj $\mathrm{S}^{3}$,Rajamani $\mathrm{G}^{4}$ \\ ${ }^{1,2,3,4}$ (Department of Paediatric Surgery, Coimbatore Medical College Hospital , Coimbatore , India)
}

\begin{abstract}
Thoracoscopic thymectomy which has a steeper learning curve is now becoming a reality in many centers in developing countries. The aim of the study is to highlight the advantage of thoracoscopic thymectomy over conventional methods in a minimal resource setup. Case records of patients diagnosed as anterior mediatinal masses from may 2006 to may 2016 were reviewed and those pertaining to diagnosis of thymus were Retrospectively analysed. Study was done in the department of pediatric surgery, Coimbatore medical college. We had twelve cases of thymic lesions out of which six were operated by median sternotomy approach and by supraclavicular approach. Our last five cases were operated thoracoscopically. Diagnoses of all these 5 cases were preoperatively confirmed with contrast CT scan. Out of these five, three were thymoma, one case was thymic abscess and another was congenital thymic cyst.Twelve patients (10 male and 2 female children) were identified for study and their age range was between 6 to 13 years. Seven children were operated via open approach and five thoracoscopically. There was no intraoperative complication during thoracoscopy approach. The operating time ranged from 60 minutes to 98 minutes (mean - 82 minutes). The mean length of post operative hospital stay in the thoracoscopic cohort was $2.5 \pm 1.5$ day and there was no difference in ICD tube removal between open and thoracoscope cohort. Postoperatively one child developed empyema which was dealt thoracoscopically. The severity of pain and postoperative movement restriction was less in the thoracoscopic approach. Thoracoscopic thymectomy is the need of the hour in developing countries, proving to be highly effective in terms of decreased post operative hospital stay, decreased work off time for the parents. and also improved cosmesis.
\end{abstract}

Keywords: Thoracoscopy, thymoma, video-assisted thoracic surgery

\section{Introduction}

Thymic lesions in children are challenging to diagnose owing to its rarity and variation in size and radiographic appearance with age. Surgical treatment of these lesions is even more difficult owing to its proximity to great vessels and phrenic nerve demanding a meticulous approach. Thymoma associated with or without myasthenia gravis, Thymic cyst and Thymic Abscess are few indications of thymectomy in children. Approach to thymus includes trans-sternal, trans cervical and video assisted thoracoscopic approach. Ever since Talbert put thoracoscopy into its first practical use for paediatric patients ${ }^{[1]}$, it has become the standard of care for most of the thoracic lesions. Thoracoscopic thymectomy was first introduced by Sugarbaker in $1993^{[2]}$. This study is presented to share our experience in thoracoscopic thymectomy in non myasthenia gravis thymus lesions and highlight certain technical points that would aid in safe and effective thoracoscopic thymectomy surgery.

\section{Materials And Methods}

All cases diagnosed as anterior mediastinal masses from May 2006 to May 2016 in Department of Paediatric Surgery, Coimbatore medical college and hospital were reviewed and those patients with the final diagnosis pertaining to thymus, managed thoracoscopically were retrospectively analysed. This study was presented in our institutional ethical committee and approval obtained. Demographic data, presenting complaints, clinical and radiologic findings, and final diagnosis were noted for all the patients. Families of all the patients were explained about the indication and nature of surgery and consent was obtained. Operating time, post operative hospital stay duration, complications and final histopathology diagnosis of patients undergoing thoracoscopic approaches were recorded. Results were reported as percent or mean \pm standard deviation wherever appropriate.

\subsection{Patient selection:}

Although most of the thymic lesion's in pediatric population are due to myasthenia gravis, none of our cases were associated with it. All our cases were referred from paediatrics department either with symptoms related to thymus or diagnosed incidentally during evaluation for respiratory distress. We had 12 cases of thymic 
lesions out of which 6 were operated by standard open median sternotomy approach and 1 was operated by supraclavicular approach. Our last 5 cases were operated thoracoscopically. Diagnoses of all these 5 cases were preoperatively confirmed with contrast CT scan. Out of these 5 cases, 3 were thymoma , 1 case was thymic abscess and another was congenital thymic cyst.

\subsection{Technical details:}

\subsubsection{OT Setup:}

The anesthetist in the head end, the surgeon and the first assistant to the right of the patient, the nurse on the right side near foot end and the monitor placed on the left side of patient satisfying ergonomic principles of thoracoscopy

\subsubsection{Anesthesia:}

We have used double lumen endotracheal tube ventilation successfully in all our cases with decreased tidal volume and increased rate of ventilation thereby minimizing lung interference in the surgical field. Lung Isolation with bronchial blockers or double lumen catheter can be achieved which is a luxury but not a necessity.

\subsubsection{Positioning:}

Supine posture with a 10-20* left lateral tilt with the help of a rolled towel or sanbag is sufficient to approach the thymic area. True lateral position is not recommended as the apical lobe of right lung interferes in dissection of thymic area.

\subsubsection{Approach:}

The thymus can be approached either via the right or left hemithorax. We Prefer The Right Hemithorax Approach Since It Is Ergonomically Easier For A Right Handed Surgeon To do a Cranial to Caudal Dissection and the delineation of anatomy is better through right hemithorax approach.However some authors prefer left hemithorax approach for ease of dissection in aorto-pulmonary window and left pericardio phrenic angle and also for good visualization of left phrenic nerve. The side that provides better outcomes remains controversial $[3\}$.

\subsubsection{Port positioning and insufflation:}

The port positioning is not fixed and it varies from surgeon to surgeon. We prefer to use three thoracoports, single $5 \mathrm{~mm} 30^{*}$ camera through $4^{\text {th }}$ or $5^{\text {th }}$ intercostals space in the midclavicular line and two $5 \mathrm{~mm}$ working ports one through $6^{\text {th }}$ or $7^{\text {th }}$ intercostal space and the other via $2^{\text {nd }}$ or $3^{\text {rd }}$ intercostals space along anterior axillary line. (Figure 1a) Although, in some centers dual cameras with double monitors are used, it does not add any extra advantage. Initial flow rate was kept at $0.5 \mathrm{~L} / \mathrm{mt}$ and subsequently increased to $6-8 \mathrm{~L} / \mathrm{mt}$. The $\mathrm{CO} 2$ insufflation pressure was kept safely at $10 \mathrm{~mm} \mathrm{Hg}$ without any hemodynamic compromise ${ }^{[4]}$.

\subsubsection{Delineation of anatomy:}

Defining the anatomy of thymic area is important. The landmarks are phrenic nerve on either side, pericardium inferiorly, and internal thoracic vessels superiorly. The right phrenic nerve identified lateral to brachicephalic vein and coursing above Superior vena cava. (Figure $1 \mathrm{~b}$ ) The left phrenic nerve courses lateral to arch of aorta. The cephalad extent of two lobes of thymus in some cases can be upto thyroid isthmus but they can be easily and safely dissected from the mediastinum.

\subsubsection{Dissection:}

The mediastinal pleura in the thymic area is first incised either by sharp dissetion or using hook cautery. The thymic tissue is differentiated from surrounding fat by its lobulation's, texture and pale yellowwhite colour. Once the thymic tissue is identified it is essential to dissect as close to the thymic tissue as possible to avoid injury to vital structures. The thymus is covered by a thin fibrous capsule, the key is to hold this capsule with endograsper to provide traction during dissection.(Figure 1c )The cephalic portion of thymus is dissected initially with a Maryland bipolar, carefully cauterizing the thymic veins draining into innominate veins. Any error in this dissection can cause troublesome bleeding. Dissection is proceeded circumferentially and wherever thymic vessels from internal mammary artery and brachiocephalic vein are encountered they can be either clipped and ligated or cauterized using bipolar or harmonic if available. (Figure 1d, e) 

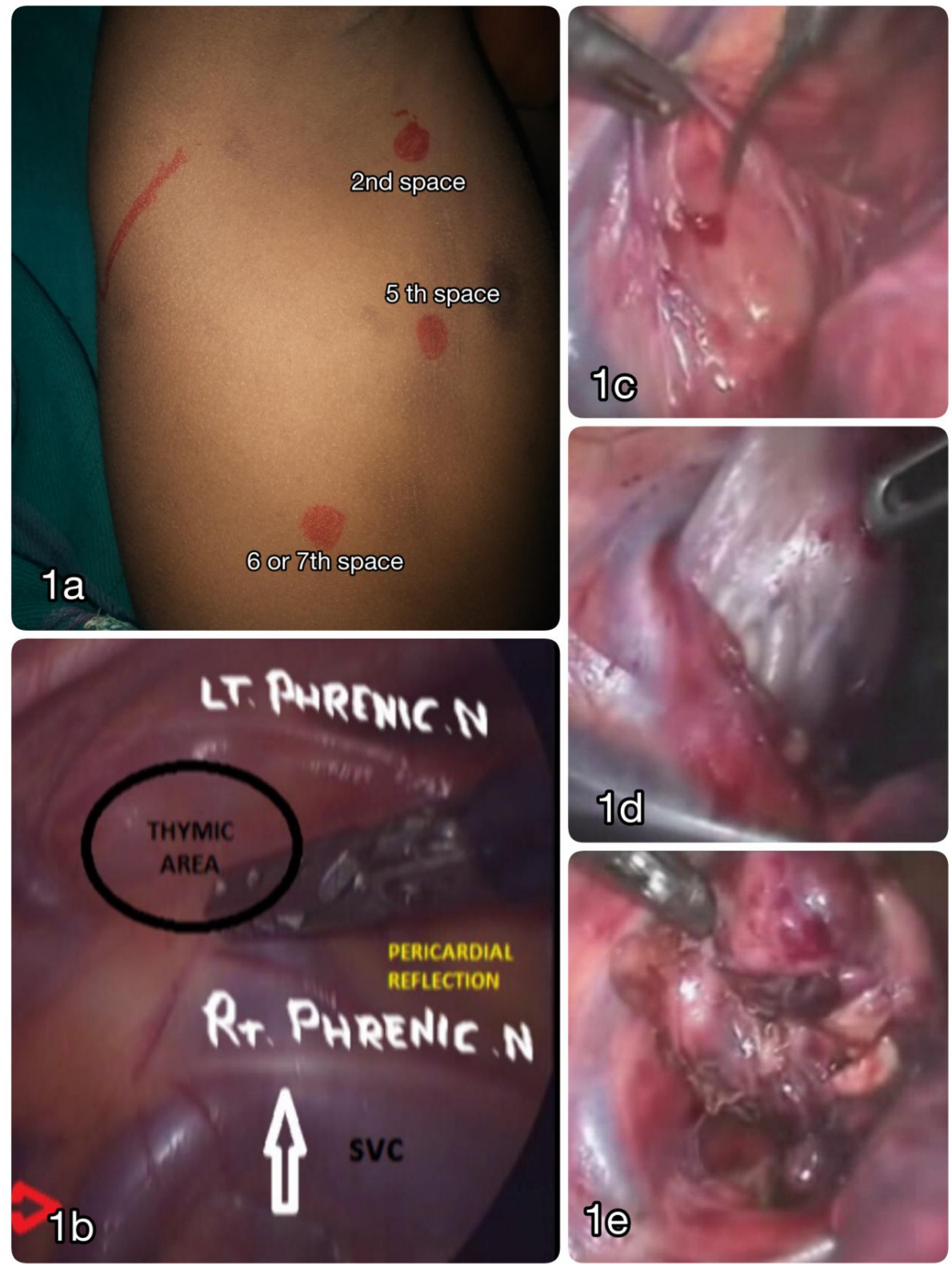

Figure 1a - port placement; 1b - thymic area boundaries; 1c- dissection of medistinal pleura; 1d-cephalad traction revealing thymic vessels; 1e-thymus circumferential dissection completed

The upper lobes are thin and can be easily pulled into mediatinum with adequate traction. The lower poles are usually thick and diffuse into pericardial fat, it is essential to remove the thymus along with this fatty tissue to clear all ectopic thymus tissue ${ }^{[5]}$. Unless the thymoma has infiltrated, there is a definite plane between thymus and anterior pericardium which is utilized during dissection. In one case of thymic abscess as the structures were densely adherent due to inflammation, the initial plane of dissection was just beneath sternum which is an avascular zone and then the abscess was drained with a suction cannula and the remaining capsule carefully dissected from adjacent structures. (Figure 2a-c)

Once the dissection is completed in the thymic area, all possible ectopic sites should be inspected and all fatty tissues should be removed. The sites to be inspected are aortocaval groove, aortopulmonary groove, left pericardiophrenic and right pericardiophrenic angles. ${ }^{[5]}$ 

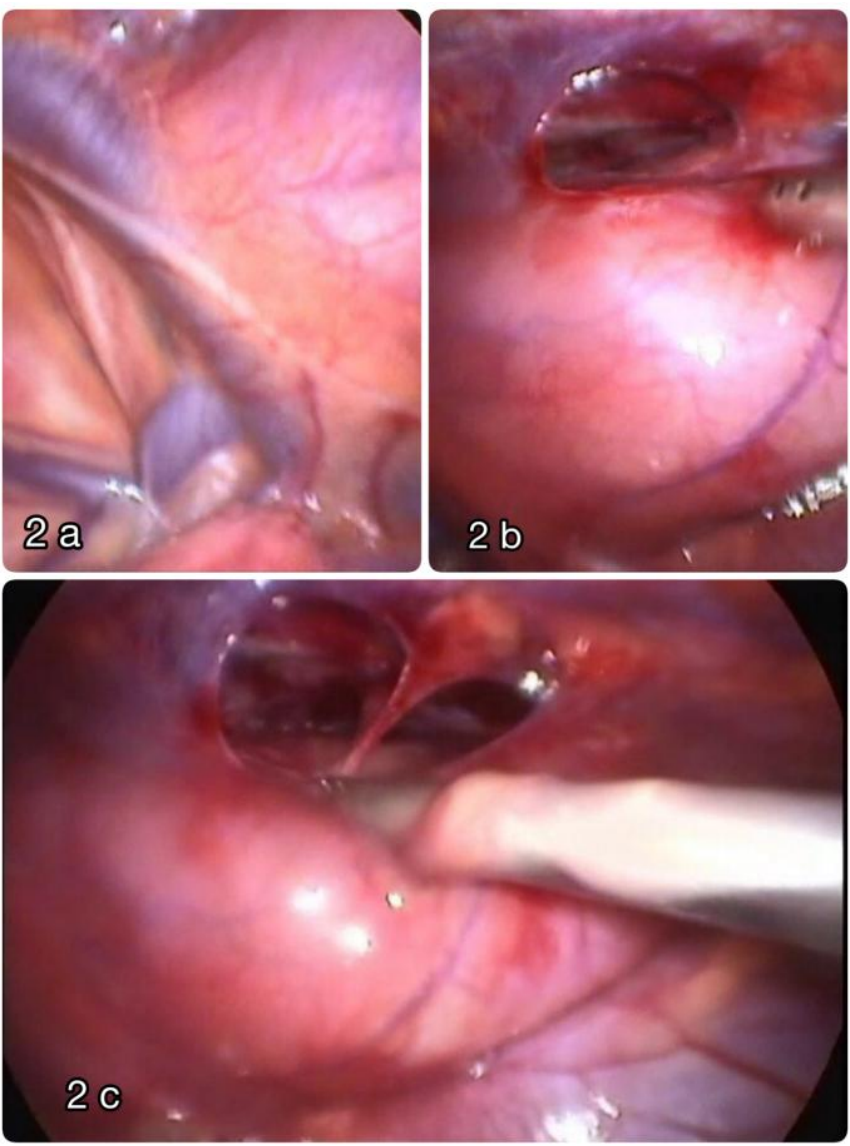

Figure 2a- thymic abscess densely adherent to SVC and phrenic nerve posteriorly;

2b- safe zone retrosternal plane created;

2c- abscess drained with suction cannula.

\subsubsection{Extraction of thymus:}

Once thymectomy is completed the specimen can be removed quite easily through the anterior most port, as anterior space is wider compared to lateral intercostals space. The specimen removed with the help of a sponge holding forceps in an endobag or modified glove bag which is a good alternative. The intercostals drainage tube is kept via 6 or 7 th space working port. Intercostal nerve block is admininstered with $025 \%$ of bupivacaine $5 \mathrm{ml}$ diluted to $10 \mathrm{ml}$.

\section{Results}

Patients who underwent thymectomy in the last ten years from May 2006 to May 2016 were included. 12 patients (10 male and two female children) were identified as a part of the study. Age range was between 6 to 13 years. 7 children were operated via open approach and 5 thoracoscopically. All the five children operated thoracoscopically were male. The cases operated thoracoscopically include 3 cases of thymoma, one case of thymic abscess and one case of thymic hyperplasia. (Table 1)

Table 1: Demographic And Clinical Details Of Thoracoscopically Managed Cases

\begin{tabular}{|l|l|l|l|l|}
\hline Age/Sex & Clinical Presentation & Procedure & Biopsy & Complications \\
\hline 7/M & Cough/Stridor & Vats - Debulking & Thymoma - B1 & Uneventful \\
\hline 6/M & Dyspnoea/Stridor & Vats- Thymectomy & Thymoma -B1 & Uneventful \\
\hline 12/M & Incidental Finding & Vats- Thymectomy & Thymic Hyperplasia & Uneventful \\
\hline 10/M & Cough/ Dyspnea & Vats - Thymectomy & Thymoma & Uneventful \\
\hline $12 / \mathrm{M}$ & Chest Pain/Fever & Vats- Drainage & Thymic Abscess & Empyema \\
\hline
\end{tabular}

There was no intraoperative complication during thoracoscopy in any of these 5 cases. The operating time ranged from 60 minutes to 98 minutes (mean 83 minutes). The mean length of post operative hospital stay in the thoracoscopic cohort was $2.5 \pm 1.5$ days. (Table 2) There was no difference in the duration of chest tube drainage postoperatively between standard open median sternotomy and between VATS thymectomy. Among the five, one case of thymic abscess developed right side empyema thoracis which was again managed 
thoracoscopically. The severity of pain and postoperative movement restriction was less in the thoracoscopic approach. However, given our small sample size this did not reach significance.

'Table 2: Post Operative Variables Comparing Open And Thoracoscopic Methods

\begin{tabular}{|l|c|c|}
\hline Variables & $\begin{array}{c}\text { Conventional } \\
\text { (Mean Days) }\end{array}$ & $\begin{array}{c}\text { Thoracoscopy } \\
\text { (Mean Days) }\end{array}$ \\
\hline Intra Op Duration & $120-140$ Minutes & $60-90$ Minutes \\
\hline Post Op Stay & $5-6$ & $2-3$ \\
\hline Icd Removal & $2-3$ & $2-3$ \\
\hline Complications & $3 / 7$ & $1 / 5$ \\
\hline
\end{tabular}

\section{Discussion}

Thoracoscopy has become the standard of care in most of the pediatric thoracic diseases like empyema $^{[6]}$, congenital lung lesions, trachea esophageal fistula, medistinal masses and thoracic duct ligation. Thymus was traditionally being approached either by median sternotomy or supraclavicular approach, with the advent of improved instrumentation and anesthesia more centers are now taking up thoracoscopic thymectomy. The results clearly demonstrate improved morbidity and better patient outcomes. Indications for thymic surgery included symptomatic patients with radiologically identified thymic lesion ${ }^{[7]}$. We did not operate on asymptomatic patients with enlarged thymus and just followed them up. In 2010 Youssef and colleagues from Seattle described their initial experience of 8 minimally invasive thymectomies and 8 traditional sternotomy thymectomies ${ }^{[8]}$. They demonstrated similar results. The length of stay for the minimally invasive thymectomies was significantly shorter at 2.4 days as compared with 4.3 days for the sternotomies $(\mathrm{P}=0.001)$. Our results are almost identical. The approaches for the minimally invasive thymectomies that Youssef et al. employed are slightly different that ours, but the end result is same. In 2013 Whitson et al demonstrated similar results with the mean length of stay significantly shorter in the VATS cohort at $2.3 \pm 1.2$ days as compared with $4.3 \pm 2.9$ days with the sternotomy approach ${ }^{[9]}$. There was no difference between approaches in the duration of chest tube drainage postoperatively. There was a trend toward less frequent complications in the VATS approach as compared with the sternotomy approach.

The leading cause of thoraoscopic thymectomy in western setup is myasthaenia gravis and thymoma ${ }^{[10]}$ . In developing country, like ours minimally invasive thymectomy remains a challenge due to less number of cases, steeper learning curve and deficient resources. But still, with maximal efforts it needs to be done in the purview of minimal postoperative complications and better cosmetic outcome ${ }^{[11-13]}$.

\section{Conclusion}

In many centers thoracoscopic thymectomy has become the standard of care even for high grade thymomas and in our study we like to emphasis that eventhough it is a technically demanding surgery, it has to be mastered as patient outcomes improve drastically with VATS. Getting used to the orientation of anterosuperor mediatinal anatomy during more commonly performed surgeries like empyema decortications or lung biopsies will help during thymectomies. Simple techniques like double lumen ventilation with less tidal volume and easily available cost effective instruments like bipolar cautery and modified glove bag is sufficient to perform thymectomy effectively in children with appreciable safety margin. With effective decrease in operating time, VATS is the need of the hour in developing countries, proving to be highly effective in terms of decreased post operative hospital stay, decreased work off time for the parents and also improved cosmesis.

\section{References}

[1]. Rodgers BM, Talbert JL. Thoracoscopy for diagnosis of intrathoracic lesions in children. J Pediatr Surg. 1976;11:703-8.

[2]. Sugarbaker DJ. Thoracoscopy in the management of anterior mediastinal masses. Ann Thorac Surg.1993;56:653-6.

[3]. Xuan Xie, Xiangfeng Gan, Baishen Chen, Zhuojian Shen, Minghui Wang, Huizhong Zhang 'et al'. Left- and right-sided videoassisted thoracoscopic thymectomy exhibit similar effects on myasthenia gravis. J Thorac Dis. 2016; 8(1): 124-132.

[4]. Wolfer RS, Krasna MJ, Hasnain JU, McLaughlin JS. Hemodynamic effects of carbon dioxide insufflations during thoracoscopy. Ann Thorac Surg. 1994; 58(2): 404-7.

[5]. Behnam Sanei et al. Distribution of mediastinal ectopic thymic tissue in patients without thymic disease. Adv Biomed Res. 2015; 4: 18

[6]. Chambers A, Routledge T, Dunning J, Scarci M. Is video-assisted thoracoscopic surgical decortication superior to open surgery in the management of adults with primary empyema?. Interact Cardiovasc Thorac Surg. 2010. Aug; 11(2):171-7.

[7]. Singla S, Litzky LA, Kaiser LR, Shrager JB. Should asymptomatic enlarged thymus glands be resected?. J Thorac Cardiovasc Surg. 2010; 140(5): 977-83.

[8]. Youssef SJ, Louie BE, Farivar AS, Blitz M, Aye RW, Vallières E. Comparison of open and minimally invasive thymectomies at a single institution. Am J Surg. 2010; 199(5): 589-93.

[9]. Bryan A.Whitson, Rafael S. Andrade, Mohi O. Mitiek, Jonathan D' Cunha, and Michael A. Maddaus. Thoracoscopic thymectomy: technical pearls to a 21st century approach. J Thorac Dis. 2013; 5(2): 129-134

[10]. Yen-Chiang Tseng, Chih-Cheng Hsieh, Hsin-Yi Huang, Chien-Sheng Huang, Wen-Hu Hsu, Biing-Shiun Huang et al, Is Thymectomy Necessary in Nonmyasthenic Patients with Early Thymoma?. J Thorac Oncol. 2013; 8(7): 952-8. 
[11]. Ashleigh Xie, Richard Tjahjono, Kevin Phan, and Tristan D. Yan. Video-assisted thoracoscopic surgery versus open thymectomy for thymoma: a systematic review. Ann Cardiothorac Surg. 2015; 4(6): 495-50.

[12]. Adnan Raza and Edwin Woo. Video-assisted thoracoscopic surgery versus sternotomy in thymectomy for thymoma and myasthenia gravis. Ann Cardiothorac Surg. 2016; 5(1): 33-3.

[13]. Nicholas R. Hess, Inderpal S. Sarkaria, Arjun Pennathur, Ryan M. Levy, Neil A. Christie, and James D. Luketich. Minimally invasive versus open thymectomy: a systematic review of surgical techniques, patient demographics, and perioperative outcomes. Ann Cardiothorac Surg. 2016; 5(1): 1-9. 